\title{
Tumor Plasticity Driven by Spatial Micro-heterogeneity in the Tumor Microenvironment Contributes to Therapy Resistance
}

\section{Theodora Fifis*}

Department of Surgery, University of Melbourne, Austin Hospital, Australia

Metastases account for over $90 \%$ of tumor related deaths. The classic anticancer inhibitors of cellular proliferation are also cytotoxic to host cells thus limiting effective doses and invariably leading to the development of drug resistance.

Recent literature demonstrate some tumors harbor mutations like the EGFR-activating mutations in lung cancer or the BRAF V600E mutation in melanoma that can be targeted with specific inhibitors with promising clinical outcomes [1,2]. Even in such cases therapy is not guaranteed for all patients as tumors cease to respond after several rounds of drug treatment either because the tumor acquires resistance due to new mutations that bypass the blocked pathway or because resistant low-frequency sub clones become dominant. The fact that no treatment eliminates all tumor cells even though the re-emerging tumors are still sensitive to the treating drugs suggests that a fraction of the tumor cells are able to evade treatment through mechanisms as yet unclear.

Factors contributing to therapy resistance include intra-tumor heterogeneity (genetic and phenotypic), tumor plasticity and spatial heterogeneity in the tumor microenvironment.

Genetic intra-tumor heterogeneity arises from accumulating tumor mutations as tumor progresses. Most of the mutations, as in normal evolution, would be expected to be neutral or deleterious to the tumor cell. Some mutations however confer survival advantages as in the examples above $[1,2]$, while other mutations may confer resistance to particular treatments. The EGFR T790M mutation for example confers resistance to EGFR tyrosine kinase inhibitors [3]. Establishing tumor mutation profiles enables better targeting of treatments.

Phenotypic heterogeneity arises in response to environmental factors during tumor progression and includes epigenetic modifications such as methylation or acetylation of genes or their associated proteins [4]. While such modifications can be clonally heritable, other phenotypic variations are transient. Local hypoxia for example activates a whole new set of genes and miRNAs resulting in tumor phenotype changes [5]. Within a solid tumor different states of tumor cell differentiation are also encountered. The majority of cells exist in the differentiated epithelial state but a sub-population of cells exists in an undifferentiated state and is known as the cancer stem cells (CSCs) [6]. Studies have shown that CSCs can be generated from epithelial tumor cells through an epithelial to mesenchymal transition (EMT) that confers both chemo resistance and invasive characteristics $[7,8]$. The CSC/mesenchymal tumor sub-population is not fixed but rather a result of tumor cell plasticity. Tumor cells transit from one state to the other in response to environmental signalling (hypoxia, therapy/ cytotoxic stress) [6]. The tumor microenvironment consists of vascular cells, fibroblasts, infiltrating immune cells, the extracellular matrix (ECM) and the signaling molecules bound to it. There is a dynamic molecular cross talk between the tumor and its microenvironment that determines tumor progression [9]. We have demonstrated significant spatial differences in vessel density and maturity between the central regions and the tumor periphery [10]. Similarly fibroblasts, infiltrating immune cells and increased expression of many tumor supporting growth factors and cytokines are associated with the perivascular regions of major vessels and the tumor periphery or invasive front, where tumor cells with CSC/mesenchymal morphology are also found [10]. Taken together, phenotypic heterogeneity within a tumor is a product of spatial heterogeneity in the tumor microenvironment.

While at any time only a very small fraction of tumor cells exist in the $\mathrm{CSC} /$ mesenchymal state, drug treatment of tumors both in culture and in vivo stimulate the transition of many more cells into a CSC/mesenchymal state [11-13]. The role of environmental spatial heterogeneity in therapy resistance has not been explored at any length. It has been suggested that tumor vessel dysfunction contribute to tumor therapy resistance due to inadequate drug delivery [14]. Experimental and histological evidence in contrast indicate that tumor cells surviving therapy treatments are found close to patent vessels and display CSC/ mesenchymal morphology $[12,15]$. Tumor cells in these regions survive treatment because they may already exist in a more resistant state and/ or because they reside in a protective environment, near patent vessels and supportive stromal cells which secrete protective growth factors/ cytokines. Thus tumor cells with the BRAF V600E mutation became resistant to the specific inhibitor when stromal HGF was present [16].

The majority of current literature is focusing on the tumor cell and driver pathways that confer resistance to tumor cells during drug treatments in cell cultures. Such studies may yield important information on tumor mutation profiles but they do not accurately reflect the situation in vivo where extrinsic signals from the tumor microenvironment contribute to the therapy outcome [16]. Therapy treatments not only impact on the tumor cells but directly and significantly influence the local tumor microenvironment [17] as well as provoking systemic host effects [18]. Cytotoxic drugs and radiotherapy administered at the maximum tolerated dose (MTD) as in cancer therapies are also cytotoxic to endothelial cells lining the tumor vessels [19]. Damaged vasculature generates an injury signal inducing a systemic healing response with a major pro-angiogenic component that benefits the surviving tumor [20]. In contrast low dose prolonged or continuous treatments such as metronomic therapies elicit tumor anti-angiogenic responses without the influx of progenitor

*Corresponding author: Theodora Fifis, Senior Research Scientist, Department of Surgery, University of Melbourne, Austin Hospital, Lance Townsend Building Level 8, Studley Rd, Heidelberg, Victoria 3084, Australia, Tel: +61394965967; Fax: +61394581650; E-mail: tfifis@unimelb.edu.au

Received December 24, 2013; Accepted February 08, 2014; Published February 13, 2014

Citation: Fifis T (2014) Tumor Plasticity Driven by Spatial Micro-heterogeneity in the Tumor Microenvironment Contributes to Therapy Resistance. J Carcinog Mutagen S8: 006. doi:10.4172/2157-2518.S8-006

Copyright: (C) 2014 Fifis T. This is an open-access article distributed under the terms of the Creative Commons Attribution License, which permits unrestricted use, distribution, and reproduction in any medium, provided the original author and source are credited. 
Citation: Fifis T (2014) Tumor Plasticity Driven by Spatial Micro-heterogeneity in the Tumor Microenvironment Contributes to Therapy Resistance. J Carcinog Mutagen S8: 006. doi:10.4172/2157-2518.S8-006

endothelial cells. In addition metronomic therapies induce antitumor immune responses and possibly directly target tumor cells, including the cancer stem cell subpopulation $[21,22]$ suggesting that they may represent better treatment options to MTD treatments.

Development of therapy resistance is a significant challenge in cancer treatments. Therapy resistance is most likely transient initially resulting from morphological changes in the tumor cells but eventually it becomes permanent. Tumor mutations, local stromal composition, and the local and systemic responses induced by the treatment, all contribute to therapy resistance. Targeting the environmental cues that regulate tumor plasticity and inhibiting the systemic host response in combination with tumor specific treatments may be the best approach for effective cancer treatments.

\section{References}

1. Chapman PB, Hauschild A, Robert C, Haanen JB, Ascierto P, et al. (2011) Improved survival with vemurafenib in melanoma with BRAF V600E mutation. N Engl J Med 364: 2507-2516.

2. Pao W, Chmielecki J (2010) Rational, biologically based treatment of EGFRmutant non-small-cell lung cancer. Nat Rev Cancer 10: 760-774.

3. Maheswaran S, Sequist LV, Nagrath S, Ulkus L, Brannigan B, et al. (2008) Detection of mutations in EGFR in circulating lung-cancer cells. N Engl J Med 359: 366-377.

4. Hatzimichael E, Crook T (2013) Cancer epigenetics: new therapies and new challenges. J Drug Deliv 2013: 529312.

5. Mimeault M, Batra SK (2013) Hypoxia-inducing factors as master regulators of stemness properties and altered metabolism of cancer- and metastasisinitiating cells. J Cell Mol Med 17: 30-54.

6. Antoniou A1, Hébrant A1, Dom G1, Dumont JE1, Maenhaut C2 (2013) Cancer stem cells, a fuzzy evolving concept: a cell population or a cell property? Cell Cycle 12: 3743-3748

7. Mani SA, Guo W, Liao MJ, Eaton EN, Ayyanan A, et al. (2008) The epithelialmesenchymal transition generates cells with properties of stem cells. Cell 133 704-715.

8. Pinto CA, Widodo E, Waltham M, Thompson EW (2013) Breast cancer stem cells and epithelial mesenchymal plasticity - Implications for chemoresistance. Cancer Lett 341: 56-62.

9. Catalano V, Turdo A, Di Franco S, Dieli F, Todaro M, et al. (2013) Tumor and its microenvironment: a synergistic interplay. Semin Cancer Biol 23: 522-532.
10. Nguyen L, Fifis T, Malcontenti-Wilson C, Chan LS, Costa PN, et al. (2012) Spatial morphological and molecular differences within solid tumors may contribute to the failure of vascular disruptive agent treatments. BMC Cancer 12: 522

11. Creighton CJ, Li X, Landis M, Dixon JM, Neumeister VM, et al. (2009) Residual breast cancers after conventional therapy display mesenchymal as well as tumor-initiating features. Proceedings of the National Academy of Sciences of the United States of America 106: 13820-13825.

12. Fifis T, Nguyen L, Malcontenti-Wilson C, Chan LS, Nunes Costa PL, et al. (20131) Treatment with the vascular disruptive agent OXi4503 induces an immediate and widespread epithelial to mesenchymal transition in the surviving tumor. Cancer Medicine 2: 595-610.

13. Xiong W, Ren ZG, Qiu SJ, Sun HC, Wang L, et al. (2010) Residual hepatocellular carcinoma after oxaliplatin treatment has increased metastatic potential in a nude mouse model and is attenuated by Songyou Yin. BMC Cancer 10: 219.

14. Danquah MK, Zhang XA, Mahato RI (2011) Extravasation of polymeric nanomedicines across tumor vasculature. Adv Drug Deliv Rev 63: 623-639.

15. Hambardzumyan D, Becher OJ, Rosenblum MK, Pandolfi PP, ManovaTodorova K, et al. (2008) PI3K pathway regulates survival of cancer stem cells residing in the perivascular niche following radiation in medulloblastoma in vivo. Genes \& development 22: 436-448.

16. Straussman R, Morikawa T, Shee K, Barzily-Rokni M, Qian ZR, et al. (2012) Tumour micro-environment elicits innate resistance to RAF inhibitors through HGF secretion. Nature 487: 500-504.

17. Junttila MR, de Sauvage FJ (2013) Influence of tumour micro-environment heterogeneity on therapeutic response. Nature 501: 346-354.

18. Daenen LG, Roodhart JM, van Amersfoort M, Dehnad M, Roessingh W, et al. (2011) Chemotherapy enhances metastasis formation via VEGFR-1 expressing endothelial cells. Cancer Res 71: 6976-6985.

19. Daruwalla J, Nikfarjam M, Greish K, Malcontenti-Wilson C, Muralidharan V, et al. (2010) In vitro and in vivo evaluation of tumor targeting styrene-maleic acid copolymer-pirarubicin micelles: Survival improvement and inhibition of liver metastases. Cancer science 101: 1866-1874.

20. Gill M, Dias S, Hattori K, Rivera ML, Hicklin D, et al. (2001) Vascular trauma induces rapid but transient mobilization of VEGFR2(+)AC133(+) endothelial precursor cells. Circ Res 88: 167-174

21. Bertolini F, Paul S, Mancuso P, Monestiroli S, Gobbi A, et al. (2003) Maximum tolerable dose and low-dose metronomic chemotherapy have opposite effects on the mobilization and viability of circulating endothelial progenitor cells. Cancer Res 63: 4342-4346.

22. Torimura T, Iwamoto H, Nakamura T, Koga H, Ueno T, et al. (2013) Metronomic chemotherapy: possible clinical application in advanced hepatocellular carcinoma. Translational oncology 6: 511-519. 